\title{
AUTOMATIC BEAM STEERING IN THE CERN PS COMPLEX
}

\author{
B. Autin, G.H. Hemelsoet, M. Martini, E. Wildner, \\ CERN, CH-1211 Geneva 23, Switzerland
}

\section{Abstract}

The recombination, transfer and injection of the four beams from the PS Booster to the PS Main Ring, have a high level of intricacy and are a subject of permanent concern for the operation of the PS Injector Complex. These tasks were thus selected as a test bench for the implementation of a prototype of an automatic beam steering system. The core of the system is based on a generic trajectory optimizer, robust enough to cope with imperfect observations. The algorithmic engine is connected to pickup monitors and corrector magnets and its decision can be validated by the operator through a graphics user interface. Automatic beam steering can only be ef®cient if the beam optics is fully con®rmed by experimental observations, a condition which forces the systematic elimination of errors both in hardware and software.

\section{INTRODUCTION}

Automatic steering in the PS complex has ${ }^{\circledR}$ rst been implemented in the transfer line TT2 which connects the $26 \mathrm{GeV}$ synchrotron (PS) to the $300 \mathrm{GeV}$ synchrotron (SPS). In this line, the beams have indeed to be carefully aligned [1] on secondary emission grid monitors for the precise measurement of the emittances which will be required for the Large Hadron Collider (LHC). The test revealed that the operation is considerably alleviated when it is aided by an ${ }^{\mathrm{a} i n t e l l i g e n t}{ }^{\mathrm{o}}$ software module and that the machine is fully reliable when the instrumentation and the correction systems respond as predicted by the classical theory of strong focusing machines [2]. In the context of the preparation of the PS for the LHC, the automation of various beam manipulations is the objective of the ABS project whose acronym stands for Automatic Beam Steering and Shaping. Among the numerous applications which are planned, priority has been given to the transfer from the $1 \mathrm{GeV}$ booster (PSB) to the PS.

The physics process is schematically represented in Fig. 1. The beams circulating in each of the four booster rings are sequentially ejected towards individual transfer channels then merged in a common line [3] and @nally injected into the PS. If no correction is applied, the momentum vectors of the four beams are different at the entry to the PS, the beams oscillate about the PS closed orbit and the beam emittances are degraded. The task of the corrections on the booster side consists of making the momentum vectors collinear. Once this is achieved, the common momentum vector is oriented tangent to the PS closed orbit by the PS injection system. The problem is much more complex than the simple alignment of a beam in a transfer line but the same correction architecture can be maintained if a generic procedure has been deßned. The data acquisition whether it comes from SEM grids or electrostatic pick-ups is the same, the correction algorithm has always to solve a linear system, the graphics interface is customized to the machine but its design is standard; the various modules are written in $\mathrm{C}$ or in Mathematica [4].

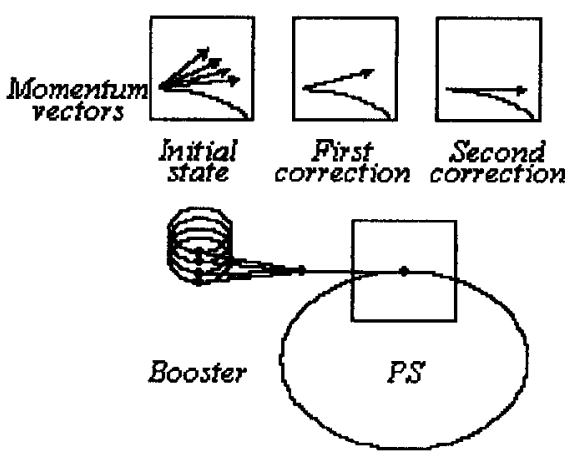

Figure 1. Transfer from PSB to PS

\section{SYMBOLIC BEAM OPTICS}

\section{A. Machine modeling}

The model of a machine is given by general purpose programs which can be numerical like $M A D$ [5] or symbolic like BeamOptics [6], a package which will be included in the PS control system as soon as a data base containing the various characteristics of the machine components is available. Some technical aspects of the application of symbolic computing to beam optics are reported elsewhere [6]. It will only be said here that functionalities such as list processing, pattern recognition, object orientation through functional programming and linkage protocols in a UNIX environment have been found very ef®cient to write concise codes and integrate them in an existing control system.

\section{B. Linear solver}

Once the theoretical model of a machine is known, its adaptation to real experimental conditions resorts to perturbation techniques, the ®rst order perturbations being by far the most frequently used. This is precisely the case of beam steering but also of tune adjustment, chromaticity, beam shaping in a ${ }^{\circledR}$ nal focus or geometrical aberrations. A $®$ rst order perturbation is linear in the sense that the total perturbation is the sum of all the individual perturbations whether the perturbation is due to a dipolar, quadrupolar, sextupolar or any type of @eld. A primary element of generality is thus to get a linear solver which can be used for any kind of problem. The Micado algorithm [7] has been selected for this purpose and its main features are brie ${ }^{-} y$ summarized.

The algorithm solves a linear system iteratively in the presence of experimental errors or even breakdowns of the instrumentation or of the correcting elements. It consists basically of minimizing iteratively the norm of a residual vector

$$
r=A x+b
$$


using a least squares method. $b$ is a vector whose $n$ components are the errors to be corrected, $x$ is the correction vector which collects the $m$ strengths to be applied to the correction magnets and $A(n, m)$ a matrix which represents the response of the beam to a set of correcting $® e l d s$.

At each iteration, the $®$ rst best magnet that yields the lowest residual r.m.s. distortion at monitor positions is selected and appended to the corrector set. Then, the residual distortion is reanalyzed and the next best magnet selected. All correctors from the previous iterations are kept but their strengths are recalculated. The method proceeds until the residual r.m.s. distortion is comparable with measurement errors.

The various steps of the calculation at the $k$-th iteration are:

1. Compute the corrector strengths as

$$
x^{(k)}=-\left(A_{i}^{(k) T} A_{i}^{(k)}\right)^{-1} A_{i}^{(k) T} b
$$

for all possible sub-matrices $A_{i}^{(k)}$ of $A$.

2. Compute the norm of the residual vector $r_{i}^{(k)}$ as

$$
\left\|r_{i}^{(k)}\right\|^{2}=b^{T} b+x_{i}^{(k) T} A_{i}^{(k) T} b
$$

3. Select the corrector which minimizes the norm of the residual vectors

$$
\left\|r_{i_{k}}^{(k)}\right\|=\min _{i}\left(\left\|r_{i}^{(k)}\right\|\right)
$$

The method is fast and converges with a small number of correctors. It has been coded as a Mathematica package.

\section{SPECIFIC ASPECTS OF BEAM STEERING}

The theory of beam steering is well known for circular machines and transfer lines. However, hybrid cases occur when the position of the beam in a transfer line is modi®ed by closed orbit distortion in an accelerator or when several transfer lines share a common section.

\section{A. Transfer line}

The correction of beam trajectories in transport channels requires a single trajectory measurement: the measurement vector is $b=\left\{u_{i}\right\}_{i=1 \ldots n}$, where $u_{i}$ is the beam position at the $i$-th pickup. The components of the correction matrix $A$ yield the trajectory deviations at the $i$-th pick-up due to a unit kick at the $j$-th corrector. For a transfer line, they are given by

$$
a_{i j}= \begin{cases}\sqrt{\beta_{i} \beta_{j}} \sin \left(\mu_{i}-\mu_{j}\right) & \text { if } \mu_{i}>\mu_{j} \\ 0 & \text { if } \mu_{i} \leq \mu_{j}\end{cases}
$$

where $\beta$ and $\mu$ are the classical $\beta$-function and phase function.

\section{B. Closed orbit}

In a ring, the orbit distortion must satisfy cyclic boundary conditions and the expression of the matrix elements is

$$
a_{i j}=\frac{\sqrt{\beta_{i} \beta_{j}}}{2 \sin \pi Q} \cos \left(-\pi Q+\left|\mu_{j}-\mu_{i}\right|\right)
$$

where $Q$ is the machine tune.

\section{Combined closed orbit and transfer line}

When the correctors of a circular machine affect the beam positions in an ejection line through a distortion of the ring closed orbit, the middle $M$ of the fast extraction kicker is taken as a hinge point. The transfer matrix from the corrector to the pick-up is then the product of the closed orbit transfer matrix from corrector to $M$ and of the channel transfer matrix from $M$ to pickup. After calculation it turns out that the expression of the correction coef®cients is formally the same as for the closed orbit correction (Eq. 6) when the origin of the phases is taken at the point $M$.

\section{Multiple transfer}

When several transfer channels are connected to a same beam line the observations in the common channel are distinct for each beam whereas the common correctors act on all the beams. This situation is tackled by de®ning two correction matrices per beam: one, called $A^{(k)}$ is attached to the individual correctors; the other one, $A^{(\mathrm{C})}$ concerns the common correctors and is the same for all the beams. The global correction matrix can thus be written

$$
A=\left(\begin{array}{ccccc}
A^{(1)} & 0 & \ldots & 0 & A^{(\mathrm{c})} \\
0 & A^{(2)} & \ldots & 0 & A^{(\mathrm{c})} \\
\ldots & \ldots & \ldots & \ldots & \ldots \\
0 & 0 & \ldots & A^{(N)} & A^{(\mathrm{c})}
\end{array}\right)
$$

\section{E. Two-turn injection}

The coherent oscillations at injection may be corrected using two successive single turn trajectories measured shortly after injection and two correction magnets per plane [8].

The trajectory position at the $i$-th pick-up in machine turn number $t$ can be expressed as

$$
u_{i, t}=z_{i}+a \sqrt{\beta_{i}} \cos \left(\mu_{i}+\varphi+2 \pi(t-1) Q\right)
$$

in which $z_{i}$ is the closed orbit position at the $i$-th pick-up. The two unknowns of the problem are the coef®cient $a$ and the constant phase $\varphi$.

The closed orbit is eliminated by taking the trajectory difference between two successive turns. The result takes the form of a classical betatron oscillation after division by $2 \sin \pi Q$.

$$
\begin{aligned}
& \frac{u_{i, t}-u_{i, t+1}}{2 \sin \pi Q}=a \sqrt{\beta_{i}} \times \\
& \quad \cos \left(\mu_{i}+\varphi+2 \pi(t-1) Q+\pi(Q-1 / 2)\right)
\end{aligned}
$$

The measurement vector $b$ in Eq. 1 is thus

$$
b=\left\{\frac{u_{i, t}-u_{i, t+1}}{2 \sin \pi Q}\right\}_{i=1 \ldots n}
$$

and the correction matrix $A$ is calculated using Eq. 5 .

When the tune is unknown, the method can still be used: the Mathematica function which selects the best correction magnets and computes their strengths, searches in addition for the tune value $Q$ which yields the minimum value of the norm of the residual vector. 


\section{EXPERIMENTAL CORRECTIONS}

\section{A. Graphics user interface}

For the operational application programs, standard graphics user interfaces have been integrated in the control system. Once the beam positions have been acquired and displayed, the operator can validate the measurements and choose the procedure of correction. The $\mathrm{C}$ program of the interface is connected to the Mathematica steering algorithm via the two way MathLink protocol. The development of the algorithm and its linkage to the control system are fully de-coupled.

An example of the organization of the interface is shown in Fig. 2 for the PS injection. The screen contains the display of the beam positions, the input to the correction algorithm and the calculated currents ready to be sent to the correction power supplies.

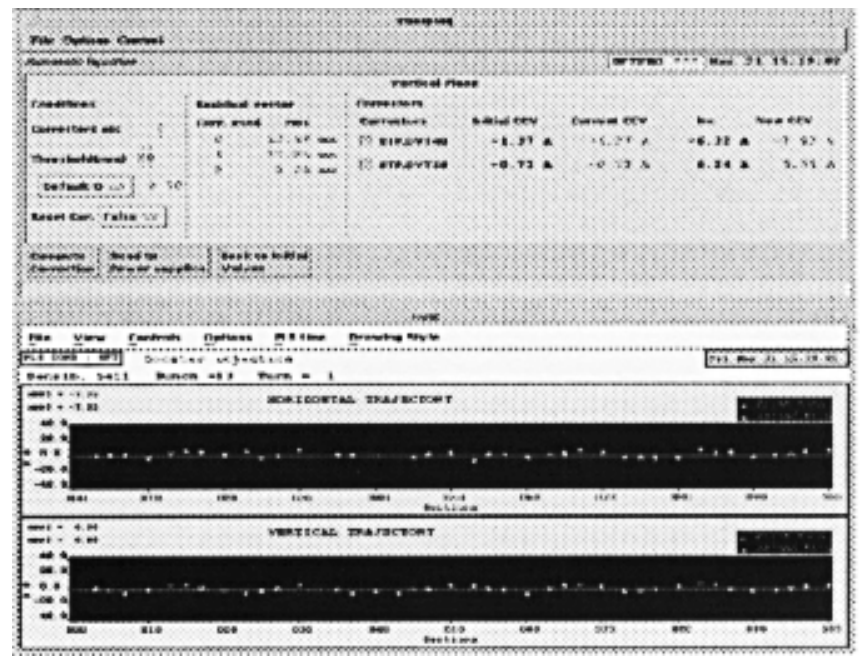

Figure 2. Graphics user interface for the PS injection

\section{B. Correction of trajectories at PSB ejection}

The procedure to correct the ejection trajectory of the PSB beam uses two dipoles in each ring and one steering magnet in the transfer line. For the vertical plane there are additional steering elements for the vertical recombination of the four rings. Downstream from the recombination point, the four beams are corrected in both planes to be aligned at the ®rst PS injection steering element.

The automation of the ejection process did not converge when it was $囚$ rst applied. A systematic error tracing was then carried out and a major system de®ciency was found to be the misalignment of the pick-ups with respect to the quadrupole axes. The measurements were ®ltered to take that misalignment into account and the correction became ef囚cient. The postion errors in each channel have typically been reduced to $2 \mathrm{~mm}$ after correction.

\section{Correction of coherent oscillations at PS injection}

The procedure has been implemented at the PS machine for the protons. The two-turn trajectories are observed with 40 pickups in each plane and there are two corrector magnets per plane. The performance of the correction procedure is closely related to the beam instrumentation. The quality of the observations has been tested by comparing the experimental calibration factor of the correctors with their values deduced from magnetic measurements. As soon as the agreement between the two determinations of the calibration factors had been obtained, the correction of the coherent oscillations became reliable and the residual amplitude was of the same order as the errors in the injection line.

\section{CONCLUSION}

An automatic beam steering procedure has been successfully implemented for the correction of the PSB ejection trajectories and of the coherent transverse oscillations at PS injection. Central in the project is symbolic computing whose resources had not yet been thoroughly investigated for accelerator controls. Computer algebra which is the best known aspect of symbolic computing is a classical tool in theoretical applications and any algorithm produced with this technique can be made available for the machine operation without any delay. At the controls level, the list processing, pattern recognition and object orientation via functional programming used to treat mathematical expressions, ${ }^{\circledR} \mathrm{t}$ the communication with equipment modules, graphics user interfaces or data bases. The experience gained on automatic beam steering in a special context will therefore be extended to other domains of the PS operation.

\section{References}

[1] B. Autin, M. Arruat, F. diMaio, M. Martini, Beam Steering: A Test Bench for Generic Algorithms in Accelerator Controls, CHEP 94, San Francisco (1994).

[2] E.D. Courant, H.S. Snyder, Theory of the AlternatingGradient Synchrotron, Annals of Physics, vol. 3, p. 1-48 (1958).

[3] J.P. Delahaye, La recombinaison des faisceaux issus des 4 anneaux du CERN PS Booster, CERN/PS/BR 79-12 (1979).

[4] S. Wolfram, Mathematica - A System for Doing Mathematics by Computer, Addison-Wesley, Redwood City, 1991.

[5] H. Grote, F.C. Iselin, The MAD Program - User's Reference Manual, CERN/SL/90-13 (AP) (1990).

[6] M. Arruat, B. Autin, G.H. Hemelsoet, M. Martini, E. Wildner, Integration of Symbolic Computing in Accelerator Control, Proc. AIHENP95, Pisa (1995), to be published.

[7] B. Autin, Y. Marti, Closed Orbit Correction of A.G. Machines Using a Small Number of Magnets, CERN ISRMA/73-17 (1973).

[8] M. Martini, J.P. Potier, T. Risselada, Automatic Injection Tuning Using Two Successive Single Turn Trajectory Measurements, Proc. EPAC 90, p. 1598-1600, Nice (1990). 